\title{
Avaliação da salinidade de Neossolo usando-se dispositivo de indução eletromagnética
}

\author{
Abelardo A. A. Montenegro', Manoel L. C. Netto ${ }^{2}$, Suzana M. G. Montenegro ${ }^{3}$, Ênio F. F. Silva ${ }^{1} \&$ Robertson V. P. Fontes Júnior ${ }^{4}$
}

\begin{abstract}
RESU MO
A concentração de sais nos solos e nas águas disponíveis em áreas irrigadas no semiárido pode comprometer a produção agrícola. Neste trabalho, desenvolvido em um vale aluvial, a salinidade do solo foi estimada a partir de leituras com 0 aparelho de indução eletromagnética EM38, considerando-se a condutividade elétrica aparente do solo (EM). Além de disposto na superfície do solo, o equipamento foi posicionado a diferentes alturas acima da superfície, de modo a incluir distintas escalas verticais na estimativa da condutividade elétrica real do solo $\left(\mathrm{CE}_{\mathrm{r}}\right)$. Modelos de regressão linear múltipla foram desenvolvidos para as condições locais e os resultados analisados através da estatística clássica e de metodologia geoestatística. As estimativas de $\mathrm{CE}_{\mathrm{r}}$ se ajustaram a uma distribuição log-Normal e apresentaram dependência espacial em escala de lote, nas camadas 0-30; 30-60 e 60-90 cm.Verificou-se que o EM 38 é uma ferramenta adequada para estimativa da salinidade do solo quando submetido a calibrações locais, em escala de lote, e de vale, mesmo em áreas com heterogeneidades verticais relevantes. 0 btiveram-se coeficientes de determinação superiores a $90 \%$ para os modelos de regressão linear múltipla.
\end{abstract}

Palavras-chave: condutividade elétrica, vales aluviais, EM 38

\section{Fluvisol salinity evaluation using electrical magnetic device}

\begin{abstract}
Salt concentration in soil and in the available waters in irrigated areas in the semi-arid regions is not always suitable, and it may interfere with the agricultural yield. In this work, soil salinity has been estimated fro $m$ readings of an electrical magnetic device EM 38 in an alluvial valley, considering the apparent soil salinity (EM). The equipment has been placed not only at the soil surface, but also at different heights above the soil level, as a way to encompass distinct vertical scales on the actual soil electrical conductivity estimation $\left(E C_{r}\right)$. Multiple regression models have been developed for the local conditions. The results have been analysed both by descriptive statistics and by the geostatistical methodology. The $\mathrm{EC}_{r}$ estimates fitted to a logN ormal distribution and presented a spatial dependence at a plot scale in the 0-30, 30-60 and $60-90 \mathrm{~cm}$ layers. It has been verified that the EM 38 is a suitable tool for soil salinity mapping, when local calibrations are considered, at plot scale, and in the valley scale, even in areas with relevant vertical heterogeneities. Determination coefficients higher than $90 \%$ has been verified for the multiple linear regression models.
\end{abstract}

Key words: electrical conductivity, alluvial valleys, EM38

\footnotetext{
${ }^{1}$ Departamento de Tecnologia Rural/UFRPE. Av. Dom Manoel de Medeiros s/n, Recife, PE, CEP 52171-900. Fone: (81) 3320-6273. E-mails: monte@hotlink.com.br; enio_silva@dtr.ufrpe.br

2 Mestre em Engenharia Agrícola. Fone: (81) 3320-6273. E-mail: manoelcosta@hotmail.com

3 Departamento de Engenharia Civil/U FPE. Av. Prof. M oraes, 1235 - Cidade Universitária, CEP 50670-901, Recife, PE, Fone: (81) 2126-7220. E-mail: suzanam@ufpe.br

${ }^{4}$ Mestrando em Engenharia Agrícola/UFRPE. Bolsista CN Pq. Fone: (81) 8605-8725. E-mail: rr_fontes@hotmail.com
} 


\section{INTRODUÇÃO}

A distribuição espacial da salinidade do solo tem grande relevância, principalmente como suporte ao manejo de água e solo em perímetros irrigados. A prevenção do processo de salinização depende de vários fatores, dentre eles a aplicação de lâminas de lixiviação que, por sua vez, são estimadas com base na salinidade real do perfil de solo na zona radicular.

A concentração de sais no solo de vales aluviais e com lençol freático raso, sofre influência de três relevantes processos: da infiltração, da ascensão capilar e dos escoamentos laterais. Consequentemente, a atuação conjunta e sucessiva desses processos nos Neossolos Flúvicos tende a gerar gradientes verticais e horizontais de salinidade, sobretudo em lotes irrigados, dificultando sua estimativa e mapeamento.

Montenegro et al. (1999) aplicaram técnicas geoestatísticas para mapeamento da condutividade hidráulica e textura de Neossolos Flúvicos do vale do Riacho Mimoso, no semiárido de Pernambuco. Devido à presença de valores discrepantes, constatou-se que a utilização de modelos robustos produziu semivariogramas mais realísticos. A variabilidade espacial da salinidade do solo subsuperficial no mesmo vale, foi analisada por Montenegro \& Montenegro (2006), que verificaram o potencial da geoestatística indicadora baseada na percentagem de silte, para identificação de áreas de maior salinidade. Para este caso não foi necessário adotar modelos robustos e a estrutura de variabilidade pode ser descrita com base no modelo clássico (Vieira et al., 1981). Por sua vez, Lyle (2007) realizou medidas de condutividade elétrica aparente com equipamento EM38 no mesmo vale observando similarmente elevada correlação entre os valores da condutividade aparente e a percentagem de silte, para profundidades de até $90 \mathrm{~cm}$. Souza et al. (2008) verificaram, em estudo em escala de lote familiar, verificaram que a condutividade elétrica do extrato saturado estava correlacionada espacialmente com o teor de silte, no Vale do Riacho Mimoso.

Corwin \& Lesch (2003) e Corwin (2005) destacam o potencial da utilização da condutividade elétrica aparente (em particular medida com o EM38) na agricultura de precisão, enfatizando sua representatividade em aplicações voltadas para estudos da variabilidade espacial da salinidade.

Para a avaliação da condutividade elétrica aparente do solo a indução eletromagnética surge como importante alternativa haja vista tratar-se de uma técnica não invasiva, que pode ser utilizada para avaliar o perfil de condutividade elétrica aparente do solo (EM), por meio de leituras múltiplas, obtidas com o instrumento disposto a diferentes alturas acima da superfície do solo, segundo McNeill (1980) e GEONICS (1999). Deste modo, as estimativas de condutividade elétrica podem ser realizadas com rapidez e praticidade e sem promover o revolvimento do solo para a retirada de amostras. O EM38, de acordo com McNeill (1980), possui altura de influência na posição vertical de aproximadamente $1,5 \mathrm{~m}$ enquanto na posição horizontal sua influência chega a $0,75 \mathrm{~m}$; desta forma, as leituras de condutividade elétrica em diferentes alturas acima do solo sofrem influência de mudanças sucessivas na salinidade com a profundidade do perfil e o alcance do equipamento (na direção vertical) sendo adequado para representar o sistema radicular da maioria dos cultivos agrícolas.

Segundo GEONICS (1999), a maior contribuição para a leitura aparente do aparelho está na camada situada a aproximadamente $40 \mathrm{~cm}$ de profundidade, para o modo vertical, e a 0 (zero) para o modo horizontal, assumindo-se o equipamento disposto na superfície do solo; assim, locais em que a leitura aparente no modo horizontal é superior à no modo vertical, tendem a apresentar perfil com maior salinidade próxima à superfície do solo.

Considerando que o EM38 fornece apenas valores aparentes e não necessariamente representativos da salinidade real, várias iniciativas têm sido desenvolvidas no sentido de relacionar a salinidade real $\left(\mathrm{CE}_{\mathrm{r}}\right)$ com as leituras aparentes (EM) do equipamento. Rhoades \& Corwin (1981) realizaram estudos para calibração do EM38, efetuando leituras (ou seja, medindo condutividades elétricas aparentes EM) com o equipamento posicionado a diferentes alturas a partir do nível do solo $(0,30,60,90,120 \mathrm{~cm})$. Com base em estimativas da condutividade elétrica real $\left(\mathrm{CE}_{\mathrm{r}}\right)$, por outros métodos, para as camadas $0-30,30-60,60-90,90-120$ e $120-150 \mathrm{~cm}$, foram desenvolvidas regressões lineares múltiplas. Coeficientes de determinação elevados foram obtidos, embora restritos às condições em que foram desenvolvidos. Na tentativa de generalização das equações de resposta do EM38, Rhoades et al. (1989) classificaram os perfis de salinidade do solo em normais ou invertidos, conforme a relação entre as leituras do EM38 no modo horizontal $\left(\mathrm{EM}_{\mathrm{h}}\right)$ e no modo vertical $\left(\mathrm{EM}_{\mathrm{v}}\right)$; assim, para $\mathrm{EM}_{\mathrm{h}}<\mathrm{EM}_{\mathrm{v}}$ os perfis eram normais e invertidos no caso contrário, razão por que se desenvolveram regressões múltiplas para estimativa da condutividade elétrica do solo $\left(\mathrm{CE}_{\mathrm{r}}\right)$ para diferentes camadas do solo em função das condutividades elétricas aparentes no modo horizontal e vertical $\mathrm{EM}_{\mathrm{h}}$ e $\mathrm{EM}_{\mathrm{v}}$. Lesch et al. (1992), por sua vez, verificaram que melhores resultados podiam ser obtidos utilizando-se a transformação do logaritmo neperiano nas leituras aparentes $\mathrm{EM}_{\mathrm{h}}$ e $\mathrm{EM}_{\mathrm{v}}$, nos modos horizontal e vertical, respectivamente. Adicionalmente, esses autores detectaram a existência de colinearidade entre as leituras nos dois modos de posicionamento e propuseram regressões considerando a diferença entre os logaritmos das condutividades aparentes, como forma de corrigir tal dependência; posteriormente, Rhoades et al. (1999) revisaram a classificação de perfil normal e invertido, com base na diferença entre os logaritmos neperianos das condutividades elétricas aparentes medidas na superfície, para um grande número de amostras.

A utilização de alturas sucessivas do equipamento acima da superfície do solo também foi adotada por Abdu et al. (2007), avaliando a sensibilidade do EM38 a variações de temperatura.

Triantafilis et al. (2000) e Yao et al. (2007) destacam a importância de se realizar calibrações e verificações locais com o EM38 e de se desenvolver modelos específicos, visto que práticas agrícolas e heterogeneidades físicas podem influenciar na distribuição de sais no perfil.

O objetivo deste trabalho é verificar o desempenho de modelos de regressão disponíveis na literatura para a esti- 
mativa da salinidade em Neossolos Flúvicos irrigados, a partir de condutividades elétricas aparentes, utilizando-se o EM38; desenvolver modelos locais de regressão para a caracterização da salinidade do solo, a partir de medidas com o EM38; e avaliar a distribuição espacial da condutividade elétrica real na zona radicular de Neossolo Flúvico.

\section{MATERIAL E MÉTODOS}

\section{Descrição do domínio}

O estudo foi desenvolvido no Agreste Setentrional de Pernambuco, região de clima semiárido, no município de Pesqueira, em áreas de assentamento rural do Estado, e com agricultura familiar, em particular na Fazenda Nossa Senhora do Rosário. As atividades de campo foram realizadas no período de abril a julho de 2007, que corresponde ao período chuvoso. O domínio de estudo está localizado na Bacia do Rio Ipanema, em particular no vale aluvial do Mimoso.

O vale do Riacho Mimoso está localizado nas coordenadas geográficas $08^{\circ} 24^{\prime} 11^{\prime \prime}$ de latitude Sul e $37^{\circ} 48^{\prime}$ '54" de longitude Oeste. A Fazenda Nossa Senhora do Rosário possui cerca de 110 ha localizados sobre aquífero aluvial, com profundidade média em torno de $10 \mathrm{~m}$, cerca de $15 \mathrm{~km}$ de extensão e $300 \mathrm{~m}$ de largura (Montenegro et al., 2003), que constitui fonte de suprimento de água para agricultura.

\section{Amostragem e análises}

Duas áreas no vale denominadas área 1 e área 2, diferindo quanto ao tipo de solo, cobertura vegetal, salinidade e uso agrícola, foram selecionadas para amostragens e medições da condutividade elétrica aparente, segundo uma malha regular de $5 \times 5 \mathrm{~m}$. Adicionalmente, leituras (seguidas de amostragem) ao acaso foram procedidas ao longo do vale, próximo a piezômetros de monitoramento (denominada área 3); no momento das leituras a área 1 estava em pousio. $\mathrm{O}$ solo é de textura franco arenosa, com textura média de 46, 28,9 e $25 \%$, de areia, silte e argila, respectivamente, com salinidade moderada, superior à da área 2; esta última possui texturas médias de 63,4,16,7 e 19,9\%, de areia, silte e argila, respectivamente e nela um experimento com a cultura da mamona irrigada, consorciada com feijão, estava em desenvolvimento. Em junho de 2007 foram coletadas amostras deformadas de solo nas distintas regiões; em cada locação, cinco amostras do solo foram obtidas nas camadas de profundidades: 0-30; 30-60; 60-90; 90-120 e 120-150 cm.

As amostras de solo foram analisadas para determinação da salinidade utilizando-se o método da pasta saturada, conforme escrito por Richards (1954) e EMBRAPA (1997), e as umidades corrigidas com base no método gravimétrico.

\section{Indução eletromagnética}

As medidas com o EM38 foram efetuadas nos modos horizontal e vertical, com o equipamento posicionado a diferentes alturas em relação a nível do solo $(0,30,60,90$, $120 \mathrm{~cm}$ ), conforme recomendado por Rhoades \& Corwin (1981). Incluiu-se, também, a altura de $150 \mathrm{~cm}$.

Fazendo-se leituras no modo vertical e horizontal em diversas alturas, pode-se chegar a um sistema de equações que permita avaliar o perfil de condutividade elétrica real $\left(\mathrm{CE}_{\mathrm{r}}\right)$ do solo.

\section{Funções de regressão}

Neste estudo se avaliaram as seguintes funções de regressão entre a condutividade elétrica do solo e leituras do dispositivo de indução eletromagnética, disponíveis na literatura:

a) Funções, segundo Rhoades \& Corwin (1981):

Dadas pela Eq. 1:

$\mathrm{CE}_{1003}=-0,1285 \mathrm{EM}_{0}+0,1446 \mathrm{EM}_{1}+5,3878 \mathrm{EM}_{2}-17,4476 \mathrm{EM}_{3}+15,0549 \mathrm{EM}_{4}-0,1309$ (1a)

$\mathrm{CE}_{\mathrm{n} 30.06}=-1,3259 \mathrm{EM}_{0}+4,8938 \mathrm{EM}_{1}+55,8250 \mathrm{EM}_{2}-94,0405 \mathrm{EM}_{4}+47,4196 \mathrm{EM}_{4}-0,9169$ (1b)

$\mathrm{CE}_{\mathrm{E} 0.699}=9,1705 \mathrm{EM}_{0}-8,4116 \mathrm{EM}_{1}-18,3090 \mathrm{EM}_{2}+50,6298 \mathrm{EM}_{3}-42,5033 \mathrm{EM}_{4}-0,1224$ (1C)

$\mathrm{CE}_{\mathrm{n}(6,090}=1,1090 \mathrm{EM}_{0}+0,2352 \mathrm{EM}_{1}-23,3536 \mathrm{EM}_{2}+221,0100 \mathrm{EM}_{3}-266,8789 \mathrm{EM}_{4}-3,5012$ (1d)

em que os índices $0,1,2,3$ e 4 se referem às alturas do EM acima da superfície do solo (0, 30, 60, 90 e $120 \mathrm{~cm})$.

b) Funções, segundo Rhoades et al. (1989):

Tais Eq. (2) estão baseadas na transformação de raiz quarta das leituras horizontais e verticais, considerando-se o equipamento posicionado apenas na superfície do solo e estão apresentadas na Tabela 1.

c) Funções, segundo Rhoades et al. (1999):

Rhoades et al. (1999) desenvolveram relações lineares entre o logaritmo neperiano de $\mathrm{EM}_{\mathrm{H}}$ e a diferença entre (ln $\mathrm{EM}_{\mathrm{H}}-\ln \mathrm{EM}_{\mathrm{V}}$ ), de modo a remover a colinearidade entre a leitura horizontal e vertical do EM38, detectada por Lesch

Tabela 1. Conjunto de equações propostas por Rhoades et al. (1989) para estimativa de condutividade elétrica do solo

\begin{tabular}{|c|c|c|c|c|}
\hline Camada (cm) & Equação para condutividade elétrica & $\mathbf{n}$ & $\mathbf{R}^{2}$ & Eq \\
\hline \multicolumn{5}{|c|}{ Para $\mathrm{EM}_{\mathrm{h}} \leq \mathrm{EM}_{\mathrm{v}}$} \\
\hline $0-30$ & $\mathrm{CE}_{\mathrm{r}}^{0,25}=2,539 \mathrm{EM}_{\mathrm{h}} 0,25-1,413 \mathrm{EM}_{\mathrm{v}}{ }^{0,25}-0,068$ & 759 & 0,810 & (2a) \\
\hline $0-60$ & $\mathrm{CE}_{\mathrm{r}}^{0,25}=2,092 \mathrm{EM}_{\mathrm{h}}{ }^{0,25}-0,81 \mathrm{EM}_{\mathrm{v}}{ }^{0,25}-0,179$ & 761 & 0,895 & (2b) \\
\hline $30-60$ & $\mathrm{CE}_{\mathrm{r}}^{0,25}=1,894 \mathrm{EM}_{\mathrm{h}}{ }^{0,25}-0,407 \mathrm{EM}_{\mathrm{v}}{ }^{0,25}-0,292$ & 758 & 0,840 & (2c) \\
\hline \multicolumn{5}{|c|}{ Para $\mathrm{EM}_{\mathrm{h}}>\mathrm{EM}_{\mathrm{v}}$} \\
\hline $0-30$ & $\mathrm{CE}_{\mathrm{r}}^{0,25}=1,164 \mathrm{EM}_{\mathrm{h}}^{0,25}-0,078 \mathrm{EM}_{\mathrm{v}}^{0,25}$ & 165 & 0,922 & (2d) \\
\hline $0-60$ & $\mathrm{CE}_{\mathrm{r}}^{0,25}=0,640 \mathrm{EM}_{\mathrm{h}}{ }^{0,25}+0,568 \mathrm{EM}_{\mathrm{v}}{ }^{0,25}-0,114$ & 163 & 0,969 & (2e) \\
\hline $30-60$ & $\mathrm{CE}_{\mathrm{r}}^{0,25}=1,367 \mathrm{EM}_{\mathrm{v}}-0,209$ & 162 & 0,919 & (2f) \\
\hline
\end{tabular}


et al. (1992). Para perfis uniformes, estabeleceu-se a seguinte relação teórica:

$\ln \left(\mathrm{EM}_{\mathrm{H}}\right)-\ln \left(\mathrm{EM}_{\mathrm{V}}\right)=0,04334+0,03058 \ln \left(\mathrm{EM}_{\mathrm{H}}\right)+0,00836 \mathrm{EM}_{\mathrm{H}}^{2}$

Três tipos de perfil foram especificados com base na Eq. 3: c1) Perfil regular, quando a medida (ln $\mathrm{EM}_{\mathrm{h}}-\ln \mathrm{EM}_{\mathrm{v}}$ ) $<5 \%$ do (ln $\mathrm{EM}_{\mathrm{h}}-\ln \mathrm{EM}_{\mathrm{v}}$ ) teórico (Eq. 3);

c2) Perfil uniforme, quando a medida $\left(\ln \mathrm{EM}_{\mathrm{h}}-\ln \mathrm{EM}_{\mathrm{v}}\right)$ está no intervalo $\pm 5 \%$ do valor teórico $\left(\operatorname{lnEM}_{\mathrm{h}}-\operatorname{lnEM}_{\mathrm{v}}\right)$;

c3) Perfil invertido, quando a medida $\left(\ln _{E^{\prime}}-\ln E_{\mathrm{v}}\right)$ $>5 \%$ do valor teórico $\left(\ln \mathrm{EM}_{\mathrm{h}}-\ln \mathrm{EM}_{\mathrm{v}}\right.$ );

Modelos matemáticos, relacionando-se o tipo de perfil e o logaritmo das condutividades aparentes, foram desenvolvidos para as camadas $0-30,30-60$, e $60-90 \mathrm{~cm}$, conforme apresentado na Tabela 2, posicionando-se o equipamento na superfície do solo.

d) Funções locais para o domínio de estudo:

Desenvolveram-se, neste estudo, regressões múltiplas baseadas no logaritmo das condutividades aparentes, posicionando-se o EM38 a diferentes alturas da superfície do solo.

Adicionalmente foram adotadas tanto as leituras no modo vertical $\left(\mathrm{EM}_{\mathrm{V}}\right)$ quanto as leituras no modo horizontal $\left(\mathrm{EM}_{\mathrm{H}}\right)$, considerando-se as diferenças $\left(\ln \mathrm{EM}_{\mathrm{H}}-\ln \right.$ $\mathrm{EM}_{\mathrm{V}}$ ), de modo a eliminar as colinearidades entre $\mathrm{EM}_{\mathrm{V}} \mathrm{e}$ $\mathrm{EM}_{\mathrm{H}}$, discutidas por Lesch et al. (1992) e utilizadas também por Rhoades et al. (1999). As regressões múltiplas foram construídas através do Software Statistica (Statsoft, 2001), com a forma da Eq. 5:

$\operatorname{lnCE} E_{\mathrm{i}}=\alpha_{0}{ }^{\mathrm{i}}\left(\operatorname{lnEM}_{0}{ }^{\mathrm{H}}-\operatorname{lnEM}_{0}{ }^{\mathrm{V}}\right)+\beta_{0}{ }^{\mathrm{i}} \operatorname{lnEM}_{0}^{\mathrm{V}}+\alpha_{1}{ }^{\mathrm{i}}\left(\operatorname{lnEM}_{1}{ }^{\mathrm{H}}-\operatorname{lnEM}_{1}{ }^{\mathrm{V}}\right)$

$+\beta_{1}{ }^{\mathrm{i}} \ln E M_{1}{ }^{\mathrm{v}}+\alpha_{2}{ }^{\mathrm{i}}\left(\operatorname{lnEM}_{2}{ }^{\mathrm{H}}-\operatorname{lnEM}_{2}{ }^{\mathrm{V}}\right)+\beta_{2}{ }_{2}^{\mathrm{i}} \operatorname{lnEM}_{2}{ }^{\mathrm{v}}+\alpha_{3}{ }^{\mathrm{i}}\left(\operatorname{lnEM}_{3}{ }^{\mathrm{H}}-\operatorname{lnEM}_{3}{ }^{\mathrm{V}}\right)$

$+\beta_{3}{ }^{\mathrm{i}} \ln E M_{3}{ }^{\mathrm{V}}+\alpha_{4}{ }^{\mathrm{i}}\left(\operatorname{lnEM}_{4}{ }^{\mathrm{H}}-\operatorname{lnEM}_{4}{ }^{\mathrm{V}}\right)+\beta_{4}{ }_{4}^{\mathrm{i}} \operatorname{lnEM}_{4}{ }^{\mathrm{V}}+\alpha_{5}{ }_{5}^{\mathrm{i}}\left(\operatorname{lnEM}_{5}{ }^{\mathrm{H}}-\operatorname{lnEM}_{5}{ }^{\mathrm{V}}\right)$

$+\beta_{5}{ }^{\mathrm{i}} \ln \mathrm{EM}_{5}{ }^{\mathrm{v}}+\beta_{6}{ }^{\mathrm{i}}$

em que i representa as camadas 0-30, 30-60 e 60-90 cm e os índices $0,1,2,3$, 4 e 5 representam as alturas do equipamento acima da superfície do solo, a saber: locado na superfície, a $30,60,90$ e $120 \mathrm{~cm}$ e até a altura de $150 \mathrm{~cm}$.

\section{Variabilidade espacial}

Após análise de Normalidade das medidas de salinidade real, a função clássica para o semivariograma, conforme apresentada por Vieira et al. (1981) foi utilizada para descrever a dependência espacial da salinidade a diferentes profundidades. As semivariâncias experimentais podem ser estimadas por:

$$
\gamma(\mathrm{h})=\frac{1}{2 \mathrm{~N}(\mathrm{~h})} \sum_{\mathrm{i}=1}^{\mathrm{N}(\mathrm{h})}\left[\mathrm{z}\left(\mathrm{x}_{\mathrm{i}}\right)-\mathrm{z}\left(\mathrm{x}_{\mathrm{i}}+\mathrm{h}\right)\right]^{2}
$$

onde $\gamma(\mathrm{h})$ são as semivariâncias e $\mathrm{N}(\mathrm{h})$ o número de pares a cada distância h.

Para a modelagem do semivariograma e sua validação (pelo processo de Jack-knifing) empregou-se a ferramenta geoestatística GS ${ }^{+}$(Robertson, 1998). Utilizou-se o processo de validação cruzada, que consiste em se reavaliar, pelo estimador, os valores amostrais conhecidos, retirando-se um a um e os calculando como se não fossem conhecidos (Vauclin et al., 1983).

$\mathrm{Na}$ presença de valores discrepantes da variável de interesse a validação cruzada, baseada no ajuste do modelo teórico às semivariâncias experimentais estimadas a partir da Equação 6, é frequentemente inadequada, casos em que se pode adotar o modelo experimental de semivariograma robusto, proposto por Cressie \& Hawkins (1980) apresentado em Montenegro \& Montenegro (2006).

Quanto à magnitude da dependência espacial (DE), os semivariogramas foram classificados segundo critério proposto por Cambardella et al. (1994), no qual os mesmos podem ser considerados como de forte, moderada ou fraca dependência espacial.

\section{RESULTADOS E DISCUSSÃO}

As Figuras 1A e B apresentam o "Box-Plot" da percentagem de areia e da condutividade elétrica do extrato saturado, respectivamente, para as áreas 1,2 e 3, e nas profundidades $0-30,30-60,60-90 \mathrm{~cm}$. Pode-se verificar a presença

Tabela 2. Equaç̧ões de regressão propostas por Rhoades et al. (1999), relacionando-se número de amostras, profundidade e medições com o EM 38

\begin{tabular}{|c|c|c|c|c|}
\hline Camada (cm) & Equação preditiva & $\mathbf{n}$ & $\mathbf{R}^{2}$ & Eq \\
\hline \multicolumn{5}{|c|}{ Para perfis regulares (valores medidos $<5 \%$ do valor teórico) } \\
\hline $0-30$ & In $C E_{r}=0,414+0,985 \ln \mathrm{EM}_{H}+2,336\left(\ln \mathrm{EM}_{H}-\ln \mathrm{EM}_{\mathrm{V}}\right)$ & 650 & 0,76 & (4a) \\
\hline $30-60$ & In $C E_{r}=0,836+1,262 \ln E_{H}+1,307\left(\ln E_{H}-\ln E_{V}\right)$ & 625 & 0,75 & $(4 b)$ \\
\hline $60-90$ & In $\mathrm{CE}_{\mathrm{r}}=0,674+1,089 \ln \mathrm{EM}_{H}-0,446\left(\ln \mathrm{EM}_{H}-\ln \mathrm{EM}_{\mathrm{V}}\right)$ & 200 & 0,69 & (4c) \\
\hline \multicolumn{5}{|c|}{ Para perfis uniformes (valores medidos dentro de $5 \%$ do valor teórico) } \\
\hline $0-30$ & In $C E_{r}=0,478+1,209 \ln \mathrm{EM}_{H}+0,411\left(\ln \mathrm{EM}_{H}-\ln \mathrm{EM}_{\mathrm{V}}\right)$ & 73 & 0,81 & $(4 d)$ \\
\hline $30-60$ & In $\mathrm{CE}_{\mathrm{r}}=0,699+1,234 \ln \mathrm{EM}_{H}-0,623\left(\ln \mathrm{EM}_{H}-\ln \mathrm{EM}_{\mathrm{V}}\right)$ & 70 & 0,81 & $(4 \mathrm{e})$ \\
\hline $60-90$ & In $\mathrm{CE}_{\mathrm{r}}=0,477+1,053 \ln \mathrm{EM}_{H}-0,691\left(\ln \mathrm{EM}_{H}-\ln \mathrm{EM}_{\mathrm{V}}\right)$ & 24 & 0,81 & (4f) \\
\hline \multicolumn{5}{|c|}{ Para perfis invertidos (valores medidos $>5 \%$ do valor teórico) } \\
\hline $0-30$ & In $C E_{r}=0,626+1,239 \ln \mathrm{EM}_{H}+0,325\left(\ln \mathrm{EM}_{H}-\ln \mathrm{EM}_{\mathrm{V}}\right)$ & 56 & 0,91 & $(4 g)$ \\
\hline $30-60$ & $\ln \mathrm{CE}_{\mathrm{r}}=0,881+1,216 \ln \mathrm{EM}_{\mathrm{H}}+1,318\left(\ln \mathrm{EM}_{H}-\ln \mathrm{EM}_{\mathrm{V}}\right)$ & 55 & 0,81 & (4h) \\
\hline $60-90$ & $\ln C E_{r}=0,563+1,206 \ln E_{H}+1,641\left(\ln E_{H}-\ln E M_{V}\right)$ & 21 & 0,91 & (4i) \\
\hline
\end{tabular}


A.

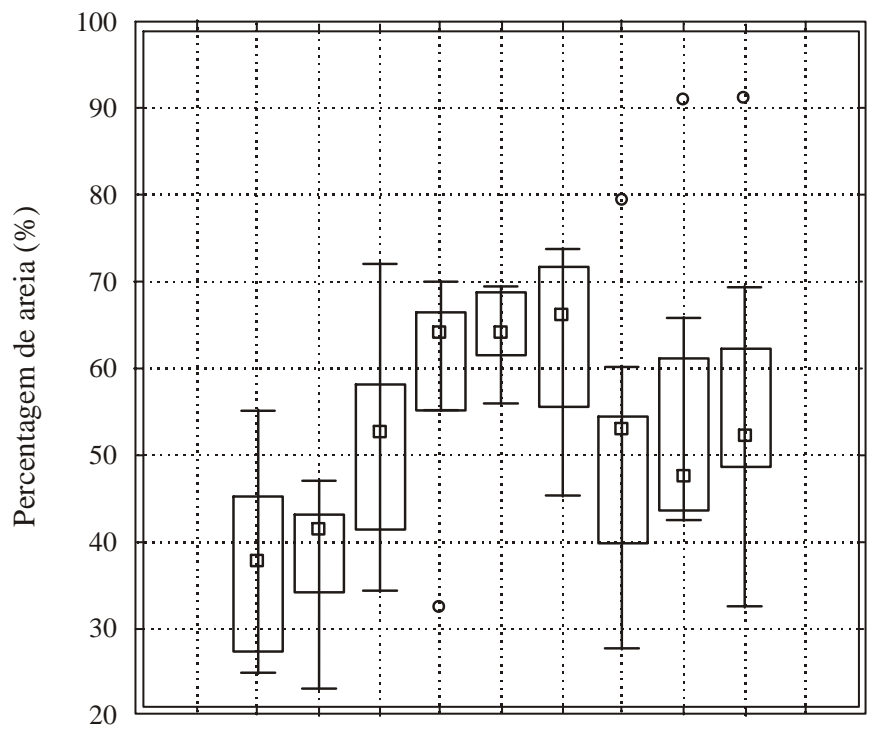

B.

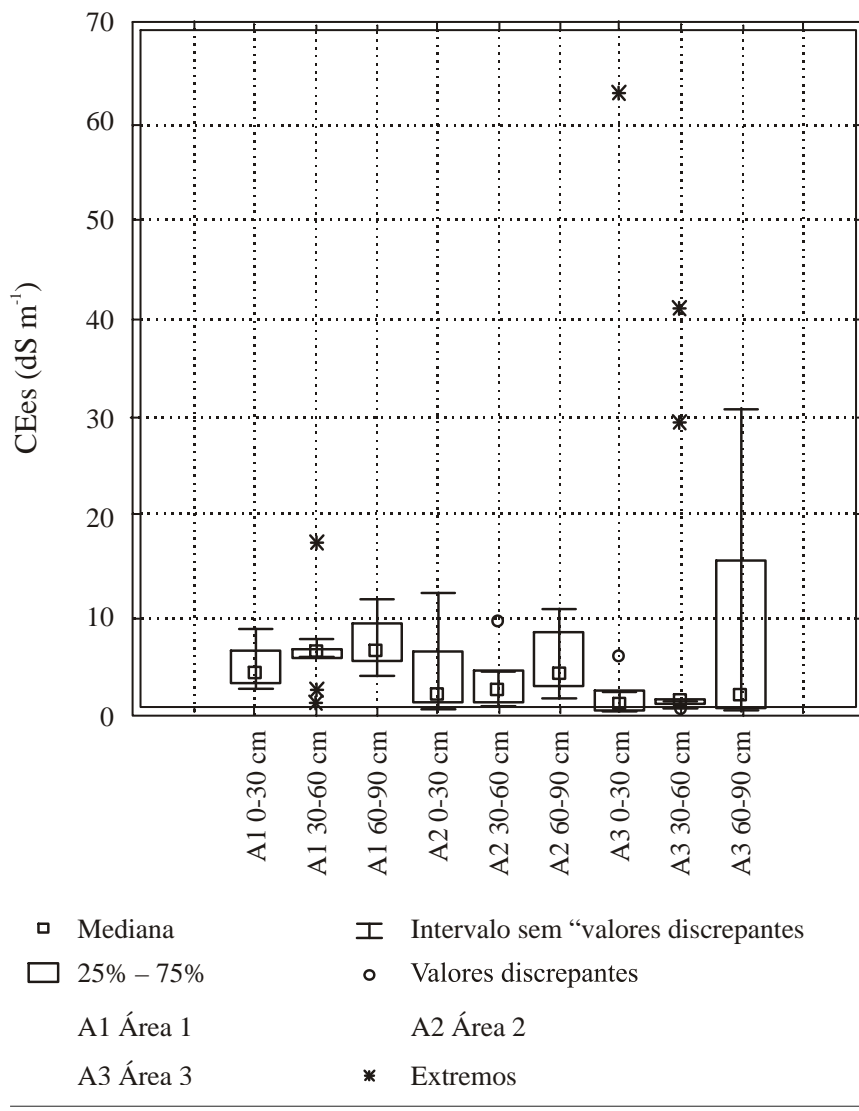

Figura 1. Percentagem de areia $(A)$ e condutividade elétrica do extrato de saturação (CEes) (B) nas áreas 1, 2 e 3, nas camadas de solo 0-30, 30-60, e $60-90 \mathrm{~cm}$ de profundidade

de perfis mais arenosos na área 2, em relação às áreas 1 e 3 , embora esta última possua valores discrepantes com solos de alto teor de areia; a área 3 também possui valores discrepantes no tocante à salinidade; nota-se, também, que a salinidade da área 2 é inferior à da área 1.

Pode-se verificar desvios-padrão elevados das condutividades aparentes na área 3 e uma relativa similaridade entre as leituras, nas áreas 1 e 2; adicionalmente, nota-se que as leituras verticais tendem a ser superiores às leituras horizontais (Figura 2).

Na Figura 3A se acham as leituras de condutividade elétrica aparente transformadas pela aplicação do logaritmo, podendo-se constatar uma colinearidade elevada entre as leituras, nos modos horizontal e vertical. A adoção da diferença $\left(\ln \mathrm{EM}_{\mathrm{H}}-\ln \mathrm{EM}_{\mathrm{V}}\right.$ ), proposta por Lesch et al. (1992), foi eficiente na remoção de tal dependência, conforme a Figura 3B.

As estimativas de condutividade elétrica real utilizandose os modelos de Rhoades \& Corwin (1981), Rhoades et al. (1989; 1999), com base nas leituras de condutividade elétrica aparente da Figura 2, estão apresentadas nas Figuras 4 e 5 , em que valores estimados estão comparados com os valores de condutividade elétrica do solo, a partir das medidas de pasta saturada e corrigidas pela umidade da amostra, conforme já descrito.

Nota-se baixo desempenho do modelo de Rhoades \& Corwin (1981), com reduzido coeficiente de determinação entre valores medidos e estimados; adicionalmente, verifica-se a ocorrência de valores estimados negativos de condutividade elétrica os quais não apresentam significado físico (Figura 4A). Embora as regressões propostas por Rhoades et al. (1989) corrijam a ocorrência de condutividades negativas, as estimativas baseadas neste modelo (Figura 4B) também apresentaram elevada dispersão nas predições, não devendo ser utilizadas para as condições da área estudada.

De modo geral, o modelo de Rhoades et al. (1999) manteve uma tendência adequada nas estimativas, em particular quando se trata de salinidade elevada tanto para as camadas de $0-30 \mathrm{~cm}$ quanto para as de $30-60$ e $60-90 \mathrm{~cm}$, conforme visualizado na Figura 5A; deve-se, entretanto, verificar que, para baixos valores de salinidade, ocorre elevada dispersão de valores e o desempenho do modelo é reduzido; em parte, tal comportamento pode ser atribuído às heterogeneidades do perfil e ao fato de que, no modelo de Rhoades et al. (1999), o equipamento é disposto apenas na superfície do solo.

A utilização de alturas adicionais tem significativa influência na redução das incertezas. Para examinar mais detalhadamente as dispersões nas estimativas das áreas com menor salinidade, as regressões locais contemplaram apenas as leituras para as áreas 1 e 2.

Apresentam-se, na Figura 5A, os coeficientes de determinação das estimativas de condutividade elétrica a partir de modelos de regressão lineares desenvolvidos especificamente para as condições deste estudo. Os coeficientes de determinação aumentam à medida em que mais alturas de posicionamento são consideradas na área 1 (valores na curva $n=9$ ) e conjuntamente para as áreas 1 e 2 (valores na curva $n=15$ ).

Como o vale aluvial em estudo apresenta solo heterogêneo, as regressões locais podem fornecer informações mais precisas devido às características qualitativas e quantitativas dos atributos físicos e químicos dos perfis, principalmente em escala de lote, conforme enfatizado por Triantafilis et al. (2000).

A Tabela 3 apresenta os coeficientes das regressões múltiplas segundo a Eq. 5 e os coeficientes de determinação 


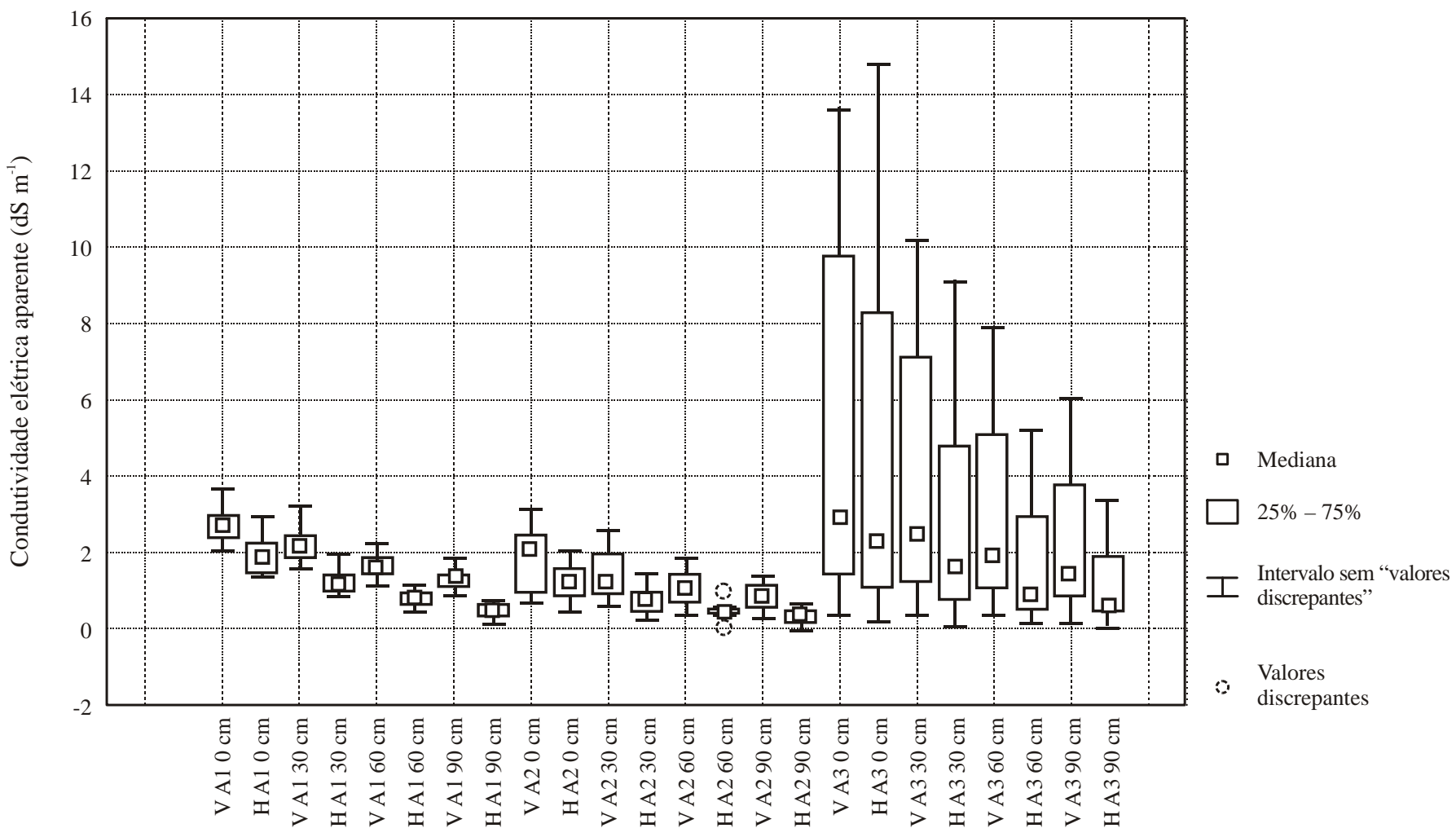

Figura 2. Condutividades elétricas aparentes nas posições vertical $(V)$ e horizontal $(H)$, com o EM 38 situado na superfície do solo, e nas alturas 30,60 e $90 \mathrm{~cm}$, nas áreas 1,2 e 3

A.

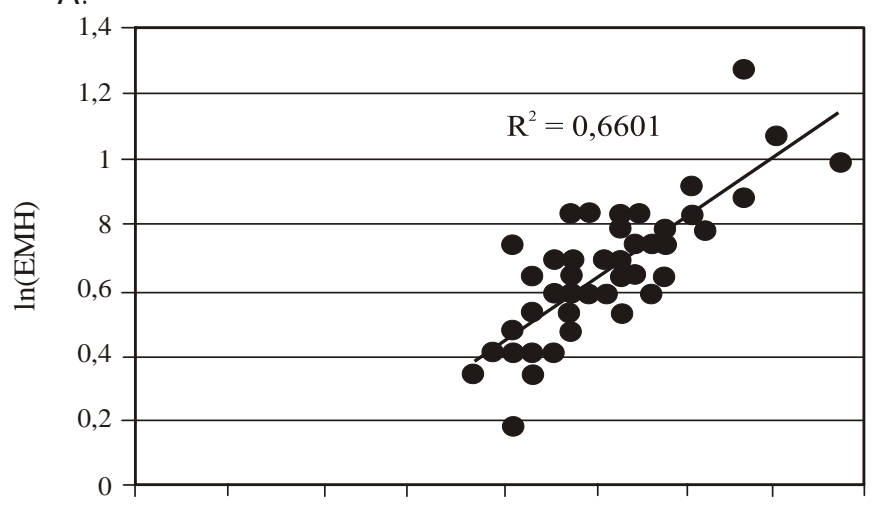

B.

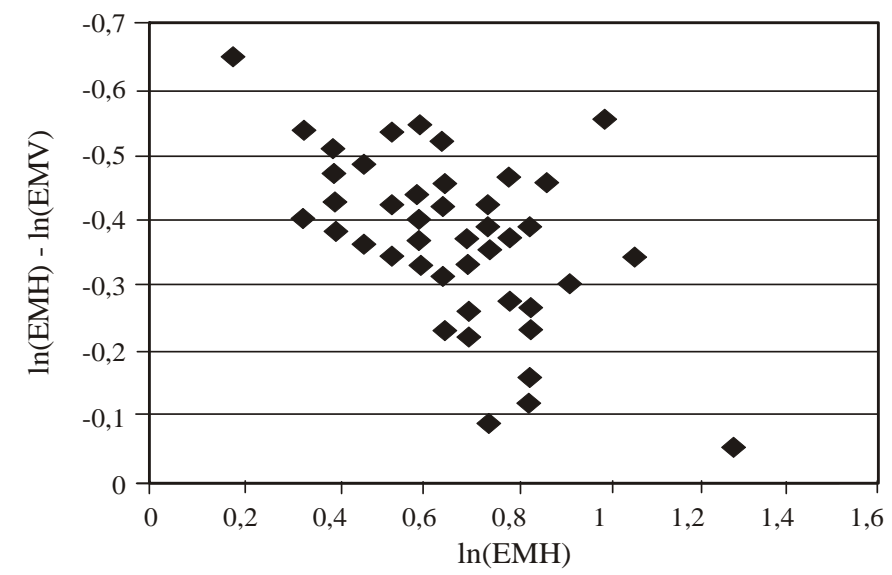

$\overline{\text { Figura 3. Relação colinear entre as leituras verticais e horizontais }(A) \text {, e }}$ remoção de colinearidade (B), para medidas aparentes de condutividade elétrica na área de estudo

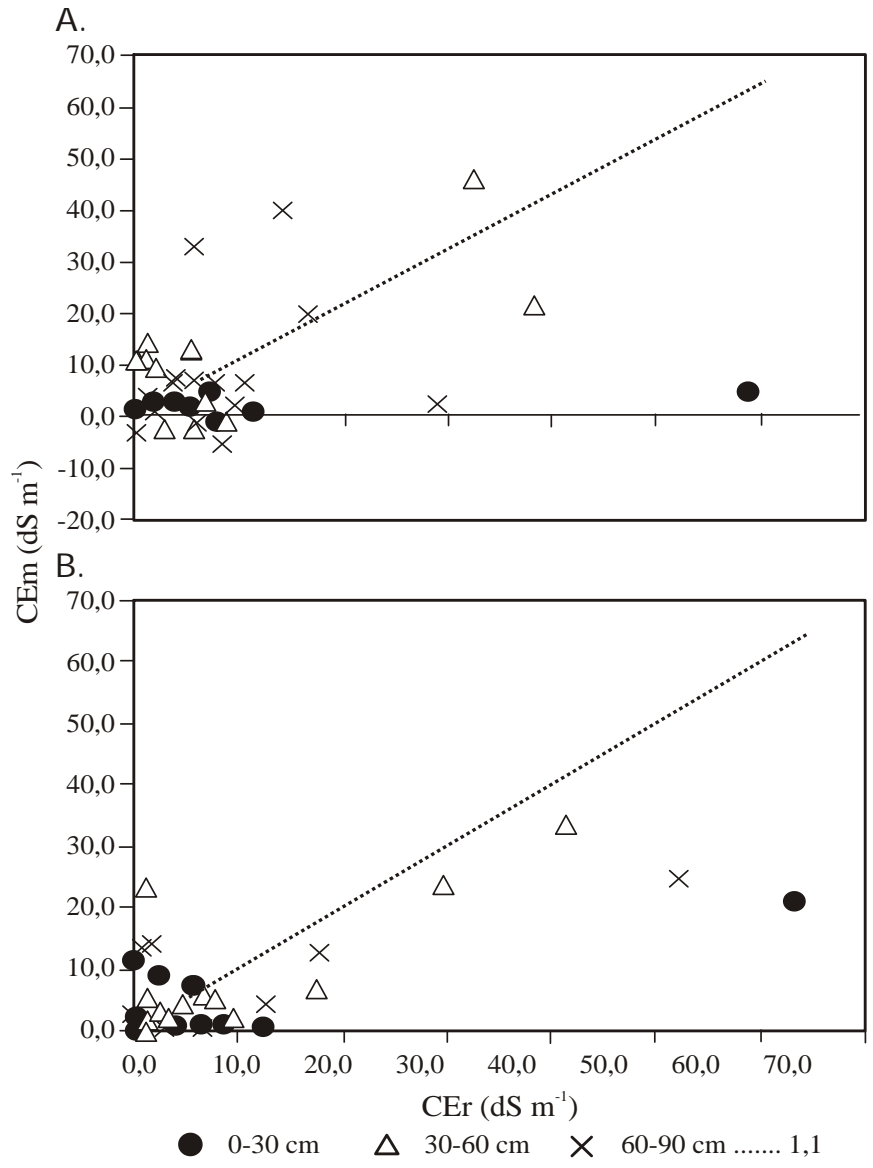

Figura 4. Condutividade elétrica real medida $\left(C E_{m}\right)$ e estimada $\left(C E_{r}\right)$, utilizando-se os modelos de Rhoades \& Corwin (1981) (A) e modelo de Rhoades et al. (1989) (B) 


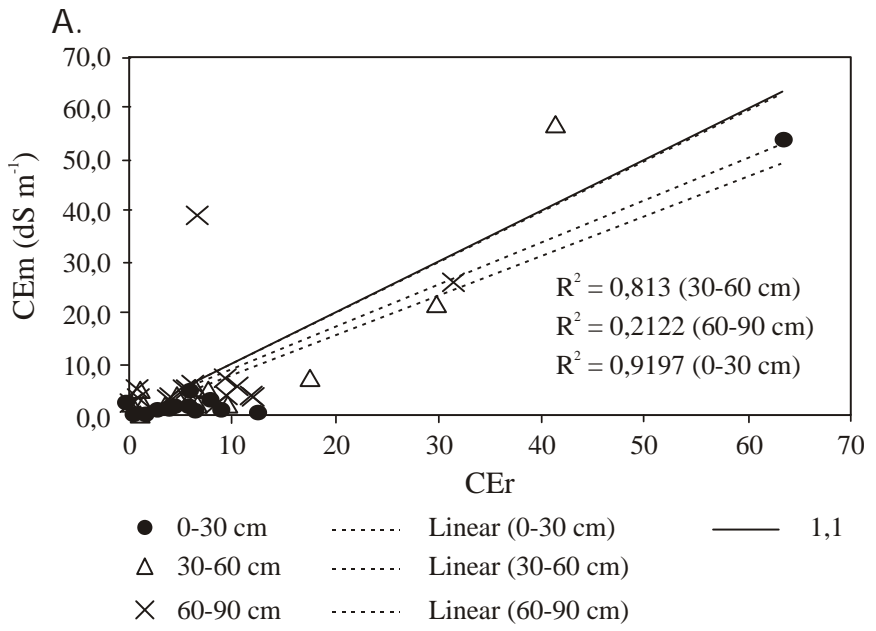

B.

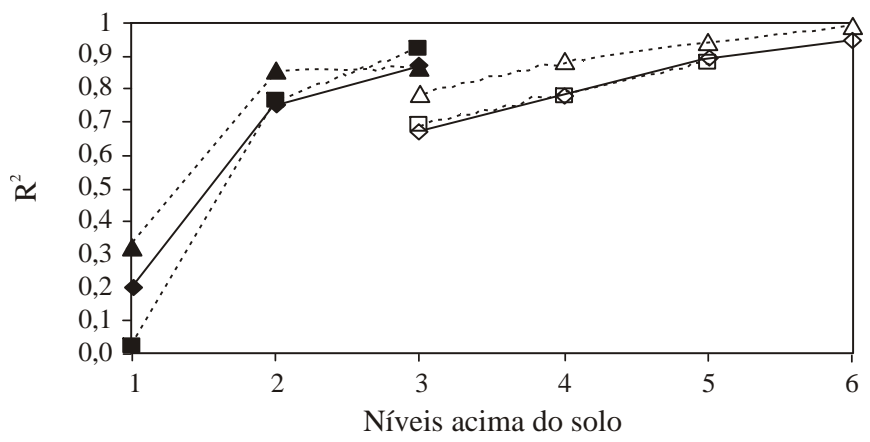

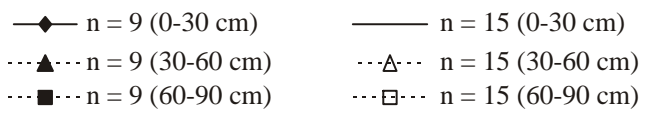

$\overline{\text { Figura 5. Condutividade elétrica real medida }\left(C E_{m}\right) \text { e estimada }\left(C E_{r}\right)}$, utilizando-se o modelo de Rhoades et al. (1999) (A); e valores de coeficiente de determinação das regressões locais, para diferentes alturas de posicionamento do EM 38 acima da superfície do solo (B)

Tabela 3. Coeficientes das regressões múltiplas locais para estimativa da condutividade elétrica real nas camadas $0-30,30-60,60-90 \mathrm{~cm}$, nas áreas 1 e 2, da Fazenda Nossa Senhora do Rosário (Eq. 5)

\begin{tabular}{rrrrrrr}
\hline & \multicolumn{2}{c}{$\mathbf{0 - 3 0} \mathbf{c m}$} & \multicolumn{2}{c}{$\mathbf{3 0 - 6 0} \mathbf{c m}$} & \multicolumn{2}{c}{$\mathbf{6 0 - 9 0} \mathbf{~ c m}$} \\
$\mathbf{R}^{2}$ & $\mathbf{0}, \mathbf{9 5}$ & \multicolumn{2}{c}{$\mathbf{0 , 9 9}$} & \multicolumn{2}{c}{$\mathbf{0 , 9 5}$} \\
$\mathbf{i}$ & $\boldsymbol{\alpha}$ & \multicolumn{1}{c}{$\boldsymbol{\beta}$} & \multicolumn{1}{c}{$\boldsymbol{\alpha}$} & \multicolumn{1}{c}{$\boldsymbol{\beta}$} & \multicolumn{1}{c}{$\boldsymbol{\alpha}$} & \multicolumn{1}{c}{$\boldsymbol{\beta}$} \\
$\mathbf{0}$ & $-2,0621$ & $-3,1814$ & 2,6417 & $-4,2511$ & $-1,6784$ & $-4,9792$ \\
1 & 4,8334 & 18,9208 & $-0,1120$ & 15,3232 & $-0,9987$ & 17,4096 \\
2 & 1,0446 & $-26,5414$ & 1,4726 & $-23,4604$ & 2,3529 & $-21,6750$ \\
3 & 1,4923 & 16,1711 & 2,4202 & 15,2102 & 0,9265 & 10,2067 \\
4 & $-0,6855$ & $-1,8629$ & $-2,6332$ & 0,5137 & $-2,9793$ & 0,1062 \\
5 & 0,2739 & $-4,1517$ & 1,2811 & $-3,0447$ & 3,1914 & $-0,6492$ \\
6 & & 1,9998 & & 3,9360 & & 2,9370 \\
\hline
\end{tabular}

encontrados para as áreas 1 e 2, simultaneamente, considerando-se o posicionamento do EM38 em 6 alturas diferentes em relação à superfície do solo.

Dentre os modelos da literatura testados neste estudo, apenas o de Rhoades et al. (1999) apresentou resultados satisfatórios, devendo, mesmo assim, ser utilizado apenas quando se deseja estudar áreas exibindo elevada amplitude de salinidade e em escala regional (vale).
Com efeito, o método de indução eletromagnética recebe influência de heterogeneidades do perfil e, deste modo, é de se esperar que o número de níveis acima do solo para posicionamento do equipamento EM38 tenha influência na redução da incerteza das regressões múltiplas, ou seja, nos coeficientes de determinação dos modelos desenvolvidos.

Pode-se observar, com base na Figura 5B, que a utilização de apenas três níveis possibilita adequado coeficiente de determinação das regressões quando se utilizam apenas as medições referentes à área $1(\mathrm{n}=9)$. Com efeito, a adoção de um único modelo local para representar a área 2 quanto a área $1(\mathrm{n}=15)$, requer que pelo menos 5 níveis de posicionamento sejam considerados. Sempre que se aumenta o número de níveis ocorre um incremento no valor do coeficiente de determinação $R^{2}$, tanto para a camada de $0-30 \mathrm{~cm}$, como para as de 30-60 e $60-90 \mathrm{~cm}$; quando se utilizam 6 níveis de posicionamento do EM 38 acima do solo, modelos de regressão com alto coeficiente de determinação podem ser desenvolvidos e com aplicabilidade simultânea para os dois lotes distintos, no vale aluvial (áreas 1 e 2).

\section{Variabilidade espacial da salinidade}

Com base nos modelos de regressão locais desenvolvidos, a variabilidade espacial da salinidade do solo na área 1 foi avaliada para o perfil de solo até a profundidade de $90 \mathrm{~cm}$. Esta região é a de maior importância no tocante ao desenvolvimento das raízes das culturas. Na Figura 6 se acham as condutividades elétricas aparentes para as cinquenta locações na área 1, cujo equipamento está disposto na superfície do solo e nas alturas 30,60 , e $90 \mathrm{~cm}$. Verifica-se que os valores dos "Box-plots" das medidas adicionais são similares ao das apresentadas na Figura 2, para a mesma área; assim, podese assumir que os modelos locais desenvolvidos são aplicáveis a este segundo levantamento; as leituras para as alturas de 120 e $150 \mathrm{~cm}$ também são similares.

Tem-se, na Tabela 4, a estatística clássica dos valores dos logaritmos da condutividade elétrica real empregando-se a Eq. 5, e os coeficientes explicitados na Tabela 3. Verificase que as condutividades elétricas seguem uma distribuição log-Normal, segundo os testes de Kolmogorov-Smirnov e Shapiro-Wilk.

Os parâmetros dos semivariogramas teóricos ajustados aos dados experimentais estão apresentados na Tabela 5 enquanto a Figura 7A exibe as semivariâncias experimentais e teóricas para as camadas 0-30, 30-60, e 60-90 cm; notase a ocorrência de semivariâncias mais elevadas na zona 30-60 cm, na qual se concentra a maior atividade das raízes, da maioria dos cultivos; adicionalmente, tal região está sujeita não só a fluxos e transporte de sais descendentes (pela precipitação pluviométrica e pela irrigação), mas também a ascendentes, provenientes de lençol freático raso no aluvião. Com efeito, durante o período de experimentos e medições a profundidade média do lençol era de $2,10 \mathrm{~m}$. Por outro lado, a maior variabilidade na camada $30-60 \mathrm{~cm}$ está em consonância com a Figura 6, tendo em vista as maiores leituras aparentes na posição vertical, que sofre maior contribuição da camada em torno de $40 \mathrm{~cm}$ de profundidade. 


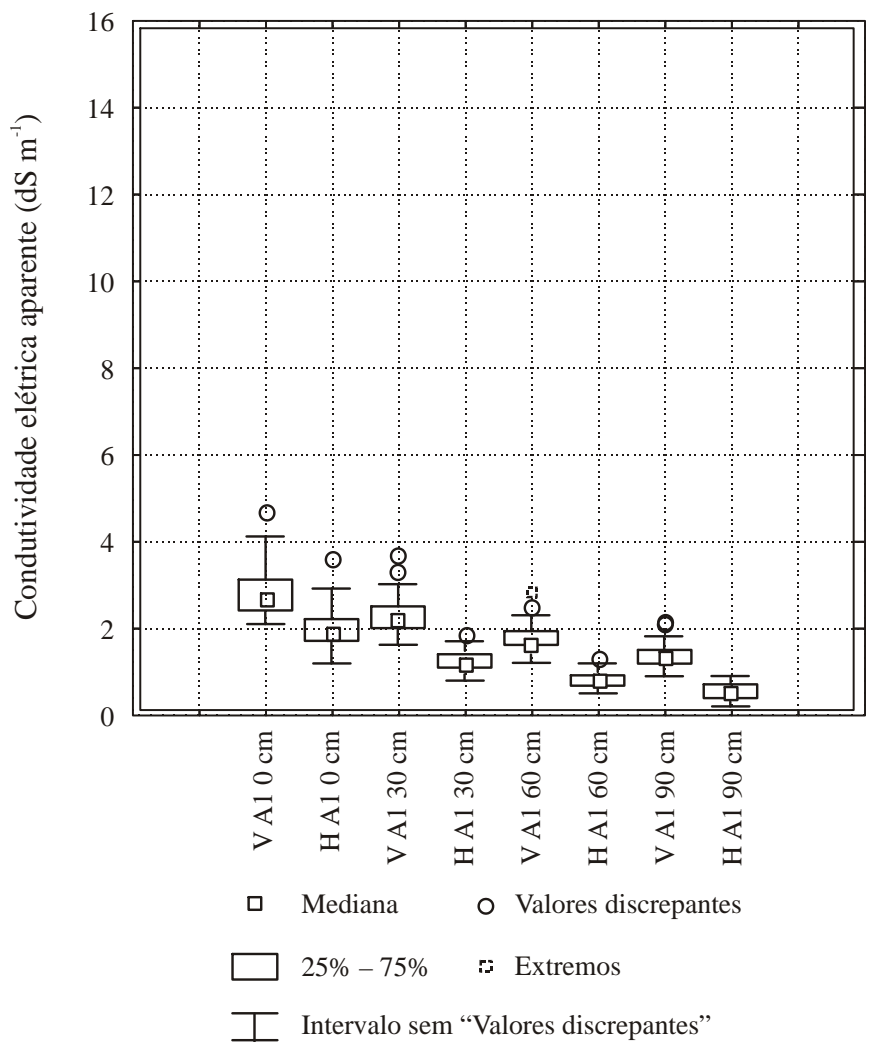

Figura 6. Condutividades elétricas aparentes nas posições vertical e horizontal, com o EM38 situado na superfície do solo e nas camadas 30, 60 e $90 \mathrm{~cm}$, na área 1

Tabela 4. Estatística clássica dos valores logaritmados de condutividade elétrica real para a área 1, nas camadas 0-30, 30-60 e 60-90 cm

\begin{tabular}{lrcc}
\hline & $\mathbf{0 - 3 0} \mathbf{~ c m}$ & $\mathbf{3 0 - 6 0} \mathbf{~ c m}$ & $\mathbf{6 0 - 9 0} \mathbf{~ c m}$ \\
Média & 1,55 & 1,72 & 1,94 \\
Erro padrão & 0,07 & 0,13 & 0,06 \\
Mediana & 1,50 & 1,76 & 1,89 \\
Variância da amostra & 0,24 & 0,78 & 0,17 \\
Curtose & 2,77 & 2,38 & 2,18 \\
Assimetria & 0,19 & 0,14 & 0,10 \\
Mínimo & 0,61 & $-0,63$ & 1,10 \\
Máximo & 3,05 & 4,04 & 2,79 \\
N & 50,00 & 50,00 & 50,00 \\
CV & 0,31 & 0,51 & 0,21 \\
\hline W (Shapiro-Wilk) & 0,98 & 0,96 & 0,98 \\
P(W) & 0,47 & 0,16 & 0,76 \\
\hline D (Kolmogorov-Smirnov) & 0,07 & 0,11 & 0,08 \\
P(KS) & $>0,20$ & $>0,20$ & $>0,20$ \\
\hline
\end{tabular}

A.

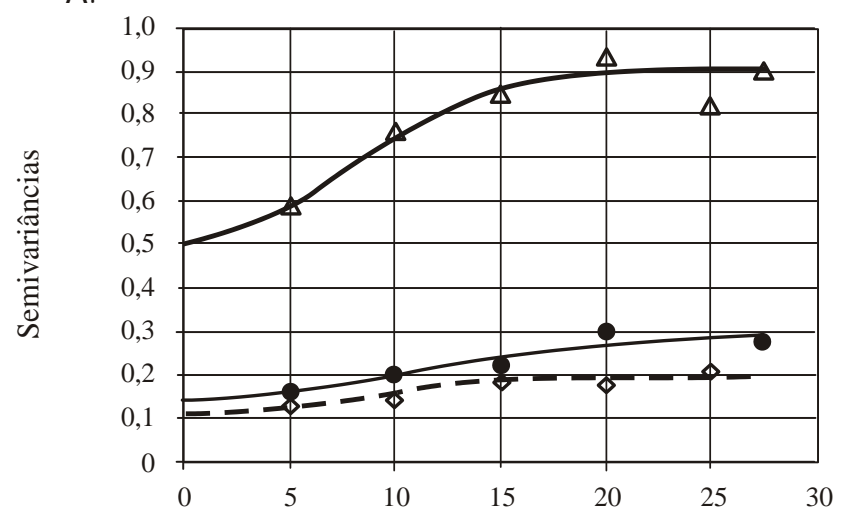

B.

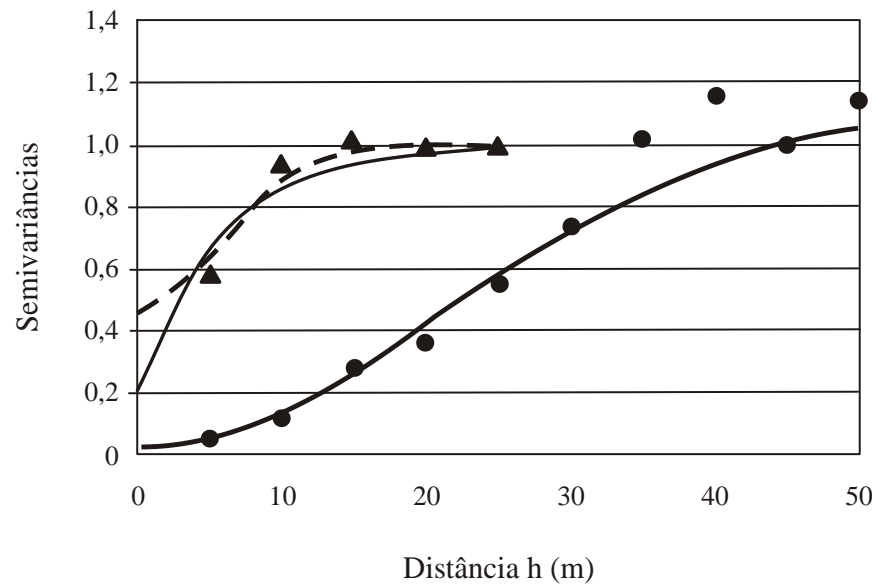

Figura 7. Semivariâncias experimentais e teóricas para In(CEr) em diferentes profundidades $(A)$, e semivariâncias cruzadas entre $\ln \left(C_{E}\right)$ e \% silte para esse estudo e do estudo de Souza et al. (2008), na profundidade $30 \mathrm{~cm}$ (B)

Tabela 5. Parâmetros dos modelos teóricos para as semivariâncias da condutividade elétrica real do solo (Teste de Jack-Knifing)

\begin{tabular}{|c|c|c|c|c|c|c|c|}
\hline \multirow[b]{2}{*}{ Camadas $(\mathrm{cm})$} & \multirow[b]{2}{*}{ Efeito pepita } & \multirow[b]{2}{*}{ Soleira } & \multirow[b]{2}{*}{ alcance } & \multicolumn{2}{|c|}{ XVALID } & \multirow[b]{2}{*}{$\mathbf{R}^{2}$} & \multirow[b]{2}{*}{$* \mathrm{DE}$} \\
\hline & & & & $\begin{array}{l}\text { Média dos } \\
\text { Resíduos }\end{array}$ & $\begin{array}{l}\text { Desvio Padrão } \\
\text { dos Resíduos }\end{array}$ & & \\
\hline \multicolumn{8}{|l|}{ Modelo Gaussiano } \\
\hline $0-30$ & 0,14 & 0,15 & 24,25 & $-0,016$ & 1,018 & 0,88 & 0,48 \\
\hline $30-60$ & 0,50 & 0,40 & 17,32 & 0,004 & 1,079 & 0,91 & 0,56 \\
\hline $60-90$ & 0,11 & 0,09 & 20,78 & $-0,016$ & 1,011 & 0,95 & 0,55 \\
\hline \multicolumn{8}{|l|}{ Modelo Exponencial } \\
\hline $0-30$ & 0,10 & 0,17 & 30 & $-0,020$ & 1,086 & 0,85 & 0,37 \\
\hline $30-60$ & 0,40 & 0,50 & 30 & 0,020 & 1,067 & 0,89 & 0,44 \\
\hline $60-90$ & 0,10 & 0,10 & 30 & $-0,080$ & 1,039 & 0,89 & 0,50 \\
\hline
\end{tabular}

*DE - Dependência espacial 


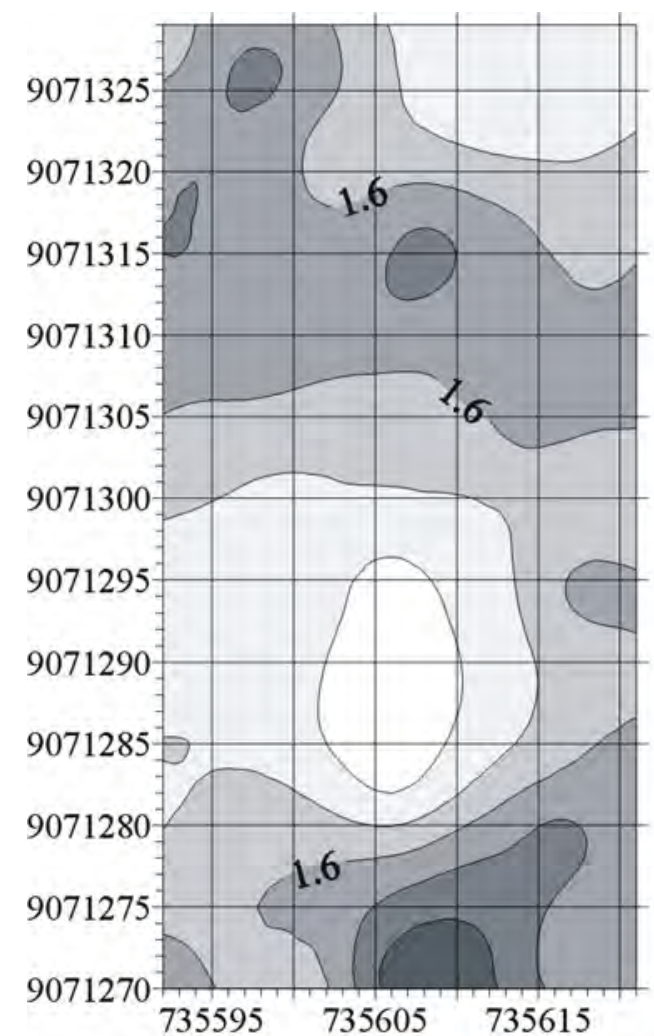

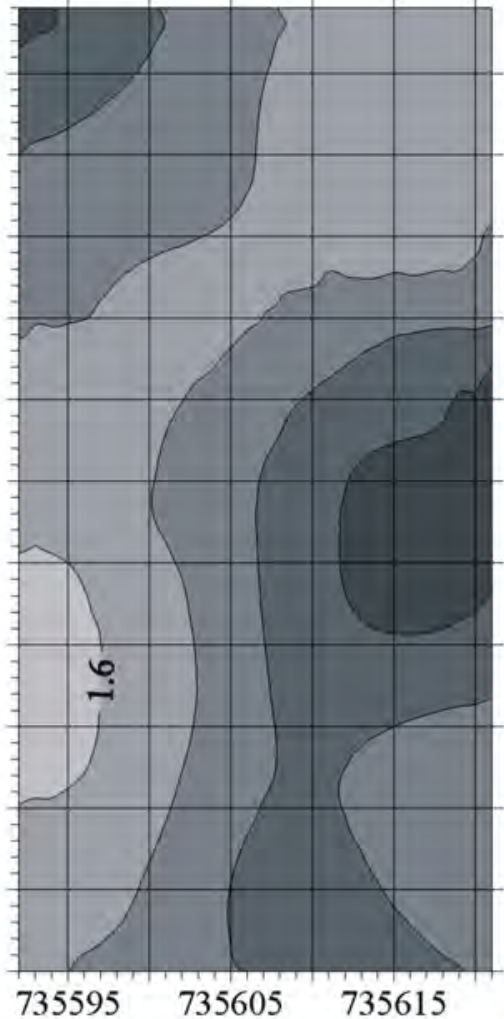

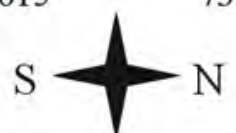

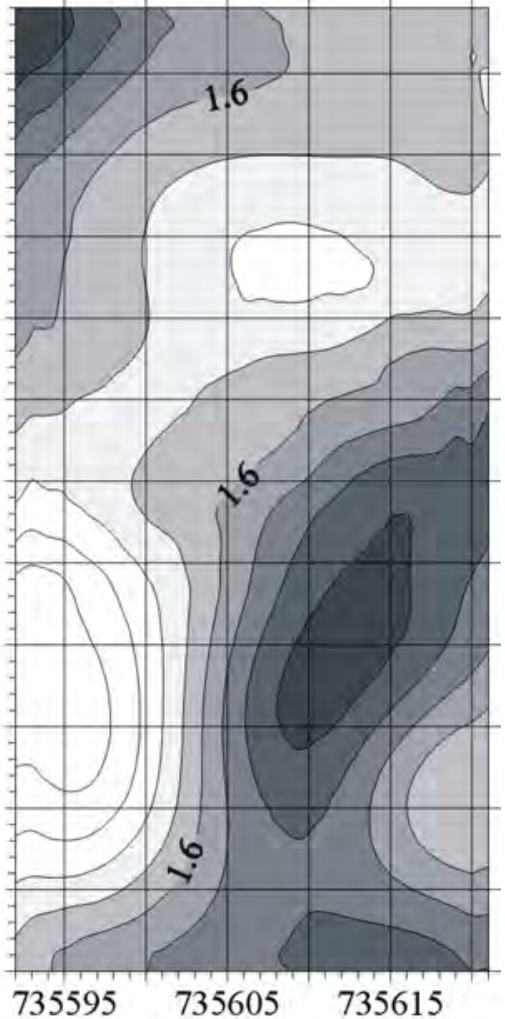

$\begin{array}{lll}735595 & 735605 & 735615\end{array}$

$\mathrm{CEr}$

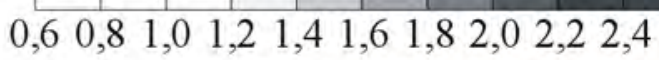

região próxima à extremidade nordeste do lote 1 .

\section{CONCLUSÕES}

1. O modelo de Rhoades et al. (1999) mostrou-se adequado para estimar condutividade elétrica do solo em áreas com elevada variabilidade de salinidade.

2. Modelos locais de regressão múltipla foram obtidos para a área em estudo, com base no posicionamento do equipamento EM38 a alturas múltiplas do solo (incluindo-se a altura de $150 \mathrm{~cm}$ ), com coeficientes de determinação superiores aos das equações disponíveis na literatura.

3. Os valores de condutividade elétrica obtidos através do EM38 apresentaram dependência espacial nas profundidades de 0-30, 30-60 cm e 60-90 cm, na área 1 , com melhor ajuste ao modelo gaussiano.

4. O conteúdo de umidade é relevante à distribuição espacial da salinidade no Neossolo considerado.

\section{AgRAdeCIMENTOS}

Os autores expressam seus agradecimentos ao suporte financeiro recebido pelo CNPq/CT-Agro, CNPq/CT-Hidro e FINEP. 


\section{LITERATURA CITADA}

Abdu, H.; Robinson, D. A.; Jones, S. B. Comparing Bulk Soil Electrical Conductivity Determination Using the DUALEM-1S and EM38-DD Electromagnetic Induction Instruments. Soil Science Society America Journal, v.71, n.1, p.189-196, 2007.

Cambardella, C. A.; Moorman, T. B.; Novak, J. M.; Parkin, T. B.; Kerlen, D. L.; Turco, R. F.; Konopka, A. E. Field scale variability of soil properties in Central Iowa Soils. Soil Science Society America Journal, v.47, p.1501-1511, 1994.

Corwin, D. L.; Lesch, S. M. Applications of soil electrical conductivity to precision agriculture: Theory, principles, and guidelines. Agronomy Journal, v.95, n.3, p.455-471, 2003.

Corwin, D. L. Applications of apparent soil electrical conductivity in precision agriculture. Computers and Electronics in Agriculture, v.46, p.1-10, 2005.

Cressie, N.; Hawkins, D. M. Robust estimation of the variogram. Journal of the International Association of Mathematical Geology, v.12, n.1, p.115-125, 1980.

EMBRAPA - Empresa Brasileira de Pesquisa Agropecuária, Serviço Nacional de Levantamento e Conservação dos Solos. Manual de métodos de análise de solo. Rio de Janeiro: EMBRAPA, 1997. 212p.

GEONICS, EM 38. Ground conductivity meter operating manual. Ontário: GEONICS Ltd. 1999. 69p.

Lesch, S. M., Rhoades, J. D., Lund, L. J.; Corwin, D. L. Mapping soil salinity unsing calibrated electromagnetic measurements. Soil Science Society America Journal, v.56, p.540-548, 1992.

Lyle, S. Application of electromagnetic techniques to investigate castor beans potential in alluvial valley of Pernambuco State. Birmingham: Earth and Environmental Sciences- University of Birmingham, 2007. 127p. Dissertação Mestrado

McNeill, J. D. Electrical terrain conductivity measurements at low induction numbers. Technical Notes TN-6, Ontario: GEONICS Ltd. 1980. 221p.

Montenegro, A. A. A.; Montenegro, S. M. G. L. Variabilidade espacial de classes de textura, salinidade, e condutividade hidráulica de solos em planície aluvial. Revista Brasileira de Engenharia Agrícola e Ambiental, v.10, n.1, p.30-37, 2006.

Montenegro, S. M. G. L.; Montenegro, A. A. A.; Mackay, R. Caracterização da variabilidade espacial de parâmetros hidráulicos em solos aluviais no Estado de Pernambuco, Revista Brasileira de Recursos Hídricos, v.4, n.2, p.5-7, 1999.
Montenegro, S. M. G. L.; Montenegro, A. A. A.; Mackay, R. Dinâmica hidro-salina em aquífero aluvial utilizado para agricultura irrigada familiar em região semiárida, Revista Brasileira de Recursos Hídricos, v.8, n.2, p.85-92, 2003.

Rhoades, J. D.; Chanduvi, F.; Lesch, S. Soil salinity assessment methods and interpretation of electrical conductivity measurements. Irrigation and Drainage, n.57. Rome: FAO, 1999. $155 \mathrm{p}$.

Rhoades, J. D.; Corwin, D. L. Determining soil electrical conductivity - depth relations using inductive electromagnetic soil conductivity meter, Soil Science Society America Journal, v.35, p.54-60, 1981.

Rhoades, J. D.; Lesch, S. M.; Shouse, P. J.; Alves, W. J. New calibrations for determining soil electrical conductivity-depth relations from electromagnetic measurements. Soil Science Society America Journal, v.53, p.74-79, 1989.

Richards, L. A. Diagnosis and improvement of saline and alkali soils. Soil and Water Conservation Ressarch Branch. Washington: Agricultural Research Service. 1954, p.159. Handbook n. 60

Robertson, G. P. Geostatistics for the environmental sciences GS+ User's Guide. Plainwell: Gamma Design Software, 1998. $152 \mathrm{p}$.

Souza, E. R.; Montenegro, A. A. A.; Montenegro, S. M. G. L.; Santos, T. E. M.; Andrade, T. S.; Pedrosa, E. R. Variabilidade espacial das frações granulométricas e de salinidade em um Neossolo Flúvico no semiárido. Ciência Rural, v.38, n.2, p.698-704, 2008.

Statsoft, Statistic: Data analysis software system. Version 6, 2001.

Triantafillis, J.; Laslett, G. M.; McBratney, A. B. Calibrating an electromagnetic induction instrument to measure salinity in soil under irrigated cotton. Soil Science Society America Journal, v.64, p.1009-1017, 2000.

Vauclin, M.; Vieira, S. R.; Vachaud, G.; Nielsen, D. R. The use of cokriging with limited field soil observations. Soil Science Society of America Journal, v.47, n.1, p.175-184, 1983.

Vieira, S. R.; Nielsen, D. R.; Biggar, J. W. Spatial variability of field-measured infiltration rate. Soil Science Society of America Journal, v.45, p.1040-1048, 1981.

Yao, R. J.; Yang, J. S.; Liu, G. M. Calibration of soil electromagnetic conductivity in inverted salinity profiles with an integration method. Pedosphere, v.17, n.2, p.246-256, 2007. 